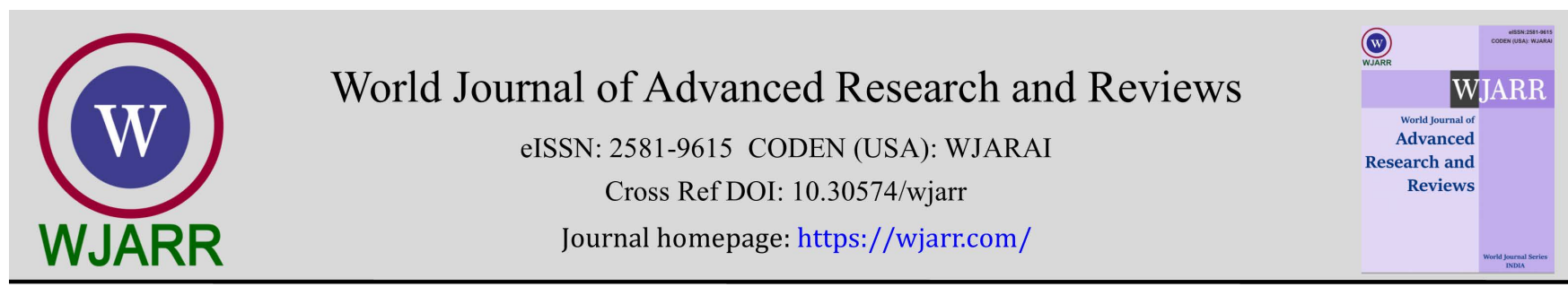

(RESEARCh ARTICLE)

Check for updates

\title{
Variability analysis of some genotypes in Nigeria tea (Camellia sinensis) germplasm
}

\begin{abstract}
Olayinka Olufemi Olaniyi ${ }^{1}$, Olusola Babatunde Kehinde ${ }^{2}$, Amos Adegbola Oloyede ${ }^{1}$, Omotayo Olalekan Adenuga ${ }^{1}$, Kayode Olufemi Ayegboyin ${ }^{1}$, Elizabeth Folasade Mapayi ${ }^{1}$, Mohammed Baba Nitsa ${ }^{1}$, Feyisetan Omolola Sulaimon ${ }^{1, *}$ and Tope Emmanuel Ebunola ${ }^{1}$
\end{abstract}

${ }^{1}$ Crop Improvement Division, Cocoa Research Institute of Nigeria.

${ }^{2}$ Federal University of Agriculture Abeokuta, Nigeria.

World Journal of Advanced Research and Reviews, 2021, 09(01), 050-061

Publication history: Received on 18 December 2020; revised on 27 December 2020; accepted on 29 December 2020

Article DOI: https://doi.org/10.30574/wjarr.2021.9.1.0491

\begin{abstract}
Thirty four tea clones were sourced from Cocoa Research Institute of Nigeria tea germplasm and raised through stem cuttings for 10 months in the nursery. The experiment was laid out in a randomized complete block design (RCBD) with 3 replications in 2016. Agronomic and yield data were collected and subjected to analysis of variance. Single linkage cluster analysis (SLCA), principal component analysis (PCA) and FATCLUS analysis were employed to analyse the data. ANOVA showed considerable significant variation $\mathrm{p}<0.05$ among the 34 tea genotypes. The PCA showed that Plant Height (PH) 0.39, Number of Leaves (NL) 0.38, Number of Branches (NB) 0.37, Harvestable Points (HP) 0.31, Stem Diameter 0.39 and Leaf Breadth 0.30 accounted for most of the variations observed. Axes 1, 2 and 3 of the PCA accounted for $37.23 \%, 15.48 \%$ and $10.75 \%$ variability respectively with cumulative value of $63.47 \%$. The genotypes were clustered into 7 groups by FASTCLUS Analysis. The dendrogram showed that the first cluster was observed between NGC29 and NGC 47 at 0.08 level of similarity. At 0.50 level of similarity the dendrogram revealed 5 distinct groups. Therefore, variation exists among the tea germplasm in Nigeria thereby suggests further presenting opportunities for further varietal development.
\end{abstract}

Keywords: Variability; Genotype; Tea; Germplasm and Analysis

\section{Introduction}

Tea (Camellia sinensis) was first cultivated in China over 2000 years ago and China remains the highest tea producer in the world [5]. It was introduced to Nigeria from Kenya in 1972 [10]. Tea is an ever green, perennial, and flowering plant belonging to the order of Theacales and family of Theaceae [21]. Commercial tea cultivars are recognized under three different taxons viz. C. sinensis, C. Assamica and C. assamicassp. lasiocalyx. Tea is a diploid ( $2 n=30$ chromosomes) but triploid and tetraploid have been reported or created [19]. It naturally grows as tall as $15 \mathrm{~m}$, but is kept between $60 \mathrm{~cm}$ and $100 \mathrm{~cm}$ under cultivation for easy harvesting, with a life span of more than 100 years [12]. It is an important crop growing across the continents of the world and major source of revenue to countries like China, Kenya, Japan, Indonesia and Vietnam. It contributes up to $26 \%$ of the Kenya annual earning [15].

Tea germplasm is considered very important for further improvement and development of new tea varieties. In the past when farming activities were still at subsistence level, natural variability was explored but now that human population is increasing at a geometric progression, there is need to be more focused on how the growing population will be fed with fixed land resources. Camellia sinensis is of high economic importance to many developing countries of the world. The sustainability of the tea industries is important owing to the fact that tea production needs to be increased to meet up with the challenges of its demand susceptibility to pest or disease. Modern breeding strategies need to be adopted

\footnotetext{
${ }^{*}$ Corresponding author: Feyisetan Omolola Sulaimon

Crop Improvement Division, Cocoa Research Institute of Nigeria.

Copyright (C) 2021 Author(s) retain the copyright of this article. This article is published under the terms of the Creative Commons Attribution Liscense 4.0.
} 
by the breeder to develop tea varieties that will combine high yielding, good cup quality and resistance to both biotic and abiotic factors to enhance its production [9].

Accurate assessment of genetic diversity of valuable crop species is a functional component of crop improvement programmes. It can also be of help for various purposes such as analysis of genetic variability of cultivars [22] and identification of diverse parental combinations to develop segregating progenies with maximum genetic variability for specific breeding purposes and further selection [6]. It is capable also for efficient germplasm management and prevention of duplicates.

The objective of the study was therefore to estimate the extent of genetic diversity and to group the 34 tea genotypes using agro-morphological characters.

\section{Material and methods}

Thirty four genotypes of tea (Camellia sinensis) were obtained from Cocoa Research Institute of Nigeria tea germplasm in Mambilla, Taraba State.

Table 1 List of the tea accessions used in the experiment.

\begin{tabular}{|l|l|l|l|l|l|l|l|l|}
\hline S/N & Genotype & Origin & S/N & Genotype & Origin & S/N & Genotype & Origin \\
\hline 1 & NGC12 & China & 13 & NGC32 & China & 25 & NGC53 & China \\
\hline 2 & NGC13 & China & 14 & NGC37 & China & 26 & NGC54 & China \\
\hline 3 & NGC15 & China & 15 & NGC38 & China & 27 & NGC55 & China \\
\hline 4 & NGC17 & China & 16 & NGC40 & China & 28 & NGC6 & China \\
\hline 5 & NGC18 & China & 17 & NGC41 & China & 29 & NGC8 & China \\
\hline 6 & NGC22 & China & 18 & NGC42 & China & 30 & NGC19 & China \\
\hline 7 & NGC23 & China & 19 & NGC46 & China & 31 & NGC45 & China \\
\hline 8 & NGC24 & China & 20 & NGC47 & China & 32 & C143 & Kenya \\
\hline 9 & NGC25 & China & 21 & NGC48 & China & 33 & C318 & Kenya \\
\hline 10 & NGC26 & China & 22 & NGC49 & China & 34 & C357 & Kenya \\
\hline 11 & NGC27 & China & 23 & NGC50 & China & & & \\
\hline 12 & NGC29 & China & 24 & NGC51 & China & & & \\
\hline
\end{tabular}

The experiment was carried out in Cocoa Research Institute of Nigeria Kusuku Mambilla sub-station, Taraba State. The plot was established in July, 2016 and Kusuku Mambilla is located on latitude 6o43' N and longitude 11o15' E. with the altitude of $1600 \mathrm{~m}$ above sea level. The type of rainfall in Mambilla is unimodal.

\subsection{Land Preparation}

The land area was cleared manually using hoe and cutlass.

Randomized Complete Block Design was used and experiment included three blocks, each containing 34 single-row plots, representing each of the tea genotypes.

Spacing of $0.6 \mathrm{~m}$ X $1.0 \mathrm{~m}$ within and between rows with plot size of $3.6 \mathrm{~m} 2$, making the total land area to be $489.6 \mathrm{~m} 2$.

The Weeding and other cultural maintenance practices were done as and when necessary. 


\subsection{Data collection}

Data were collected on 9 plants per plot in 3 months interval for 2 years using meter rule, vernier caliper and electronic weighing balance.

\subsection{Quantitative Characters Measured}

Internodes length $(\mathrm{cm})$, Length of mature leaf $(\mathrm{cm})$, Width of mature leaf $(\mathrm{cm})$, Plant height $(\mathrm{cm})$, No of branches, Shoot weight (g), Stem diameter, Number of leaves, Number of flower buds.

\section{Results}

\subsection{Analysis of variance}

Table 2 shows mean separation of the combined analysis of variance of 34 tea genotypes tested across 3 environments. The result showed that C143 was the tallest $(96.62 \mathrm{~cm})$ followed by NGC $25(88.14) \mathrm{cm}$ but not significantly different from each other. The least plant height was observed NGC 55 with average value of $34.26 \mathrm{~cm}$ and significantly different from 143 and NGC 25. NGC 38 recorded highest number of leaves 205 while the least number of leaves were observed for NGC 55 (43.18) and statistically different from each other. Number of branches and harvestable point were highest in C143 with value of 30.22 and 15.17 respectively while the least values for the two traits were observed in NGC55 with values of 6.29 and 2.40 respectively. Furthermore, C357 recorded highest stem diameter $16.83 \mathrm{~mm}$ and the least stem diameter was recorded for NGC 55 with average value of $(9.28 \mathrm{~mm})$ and significantly different from each other. It was observed that NGC 53 recorded the longest internodes length (4.45) cm and is significantly different from NGC 48 with average of $2.73 \mathrm{~cm}$. The longest leaf length was observed in NGC 50 with average of $12.92 \mathrm{~cm}$ followed by NGC 54 with value of $9.02 \mathrm{~cm}$ and statistically different from each other while the least was observed in NGC 55 (5.88cm). C357 has the largest leaf size $(4.88 \mathrm{~cm})$ and is significantly different from NGC 38 with $2.79 \mathrm{~cm}$. Genotype with the highest number of flowers was C357 with average of 41.29 flowers while the genotype with the least flower was NGC 41 with 3.44 flowers. NGC 40 recorded the highest yield /plant with average value of $10.87 \mathrm{~g} /$ plant while the least was recorded for NGC55 with average value of 0.9g/plant and significantly different from NGC40 above.

Table 3 described means among 34 tea genotypes for 3 proximate analyses viz avis protein, crude fibre and caffeine. The highest protein content observed was observed in NGC $38(22.74 \%)$ The least protein content was recorded for NGC 8 value of $18.83 \%$. The highest crude fibre content among 34 tea genotypes $10.16 \%$ recorded for NGC 40 while NGC 8 showed the lease fibre content of $8.41 \%$ and significantly different from NGC40. Finally, $2.65 \%$ caffeine content was observed in NGC 40 which was the highest and significantly different from NGC 8 with the lease value of $1.37 \%$. 
Table 2 Mean performance of 34 tea genotypes evaluated for 10 agronomic characters in Mambilla

\begin{tabular}{|c|c|c|c|c|c|c|c|c|c|c|c|}
\hline $\mathbf{S} / \mathbf{N}$ & Genotypes & $\begin{array}{l}\text { Plant } \\
\text { Height }\end{array}$ & $\begin{array}{l}\text { No. of } \\
\text { Leaves }\end{array}$ & $\begin{array}{l}\text { No. of } \\
\text { Branches }\end{array}$ & $\begin{array}{l}\text { Harvestable } \\
\text { Point }\end{array}$ & $\begin{array}{l}\text { Stem } \\
\text { Diameter }\end{array}$ & $\begin{array}{l}\text { Internodes } \\
\text { Length }\end{array}$ & $\begin{array}{l}\text { Leaf } \\
\text { Length }\end{array}$ & $\begin{array}{l}\text { Leaf } \\
\text { Breadth }\end{array}$ & $\begin{array}{l}\text { No. of } \\
\text { Flower } \\
\text { Bud }\end{array}$ & Yield/Plant(g) \\
\hline 1 & C143 & $96 \cdot 62 \mathrm{a}$ & $174 \cdot 30_{\mathrm{a}-\mathrm{f}}$ & $30 \cdot 22 \mathrm{a}$ & $15 \cdot 17 \mathrm{a}$ & $14 \cdot 93 \mathrm{a}-\mathrm{c}$ & $3 \cdot 49_{\mathrm{a}-\mathrm{c}}$ & $9 \cdot 44 b$ & $4 \cdot 16 b$ & $19 \cdot 40_{c}$ & $8 \cdot 14 b c$ \\
\hline 2 & NGC25 & $88 \cdot 14_{\mathrm{ab}}$ & $203 \cdot 62 \mathrm{a}$ & $22 \cdot 09_{b-d}$ & $7 \cdot 79_{\mathrm{e}-\mathrm{i}}$ & $15 \cdot 66_{\mathrm{ab}}$ & $3 \cdot 08 \mathrm{bc}$ & $8 \cdot 79_{b}$ & $3 \cdot 93 \mathrm{bc}$ & $8 \cdot 79_{\mathrm{f}-\mathrm{k}}$ & $5 \cdot 16_{d-h}$ \\
\hline 3 & C357 & $87 \cdot 04 a-c$ & $183 \cdot 88_{a-d}$ & $21 \cdot 06 \mathrm{~b}-\mathrm{e}$ & $11 \cdot 47_{b c}$ & $16 \cdot 83_{a}$ & $3 \cdot 71_{\mathrm{a}-\mathrm{c}}$ & $9 \cdot 44 b$ & $4 \cdot 88_{a}$ & $41 \cdot 29 a$ & $7 \cdot 00_{\mathrm{cd}}$ \\
\hline 4 & NGC49 & $79 \cdot 97_{b-d}$ & $156 \cdot 04 \mathrm{~b}-\mathrm{i}$ & $20 \cdot 68 b-e$ & $8 \cdot 00_{d-i}$ & $12 \cdot 38_{\mathrm{c}-\mathrm{k}}$ & $3 \cdot 56 \mathrm{a}-\mathrm{c}$ & $8 \cdot 83 b$ & $3 \cdot 53_{c-f}$ & $16 \cdot 46_{c-f}$ & $4 \cdot 45_{\mathrm{e}-\mathrm{l}}$ \\
\hline 5 & NGC19 & $77 \cdot 34_{\text {b-e }}$ & $206 \cdot 15 \mathrm{a}$ & $24 \cdot 12_{b}$ & $11 \cdot 50_{\mathrm{bc}}$ & $13 \cdot 05_{b-j}$ & $3 \cdot 24_{b c}$ & $8 \cdot 89 \mathrm{~b}$ & $3 \cdot 33_{\mathrm{d}-\mathrm{g}}$ & $18 \cdot 24_{\mathrm{cd}}$ & $9 \cdot 31_{\mathrm{a}-\mathrm{b}}$ \\
\hline 6 & NGC18 & $75 \cdot 82_{c-f}$ & $168 \cdot 48_{a-g}$ & $23 \cdot 50_{b c}$ & $12 \cdot 47_{\mathrm{ab}}$ & $12 \cdot 69_{c-j}$ & $3 \cdot 39_{b c}$ & $9 \cdot 29 \mathrm{~b}$ & $3 \cdot 29_{\mathrm{d}-\mathrm{g}}$ & $7 \cdot 88_{\mathrm{g}-\mathrm{k}}$ & $5 \cdot 18_{d-g}$ \\
\hline 7 & NGC27 & $75 \cdot 40_{\mathrm{c}-\mathrm{g}}$ & $141 \cdot 95_{\mathrm{e}-\mathrm{j}}$ & $17 \cdot 91_{\mathrm{c}-\mathrm{h}}$ & $7 \cdot 67 \mathrm{e}-\mathrm{i}$ & $14 \cdot 12 \mathrm{~b}-\mathrm{g}$ & $3 \cdot 86 \mathrm{ab}$ & $8 \cdot 19 \mathrm{bc}$ & $3 \cdot 28 \mathrm{~d}-\mathrm{h}$ & $14 \cdot 33_{c-h}$ & $2 \cdot 69_{\mathrm{j}-\mathrm{o}}$ \\
\hline 8 & NGC13 & $74 \cdot 38_{\mathrm{d}-\mathrm{g}}$ & $142 \cdot 96_{\mathrm{d}-\mathrm{j}}$ & $19 \cdot 09_{\mathrm{b}-\mathrm{g}}$ & $10 \cdot 15 \mathrm{~b}-\mathrm{e}$ & $14 \cdot 59_{\mathrm{a}-\mathrm{d}}$ & $3 \cdot 59_{\mathrm{a}-\mathrm{c}}$ & $9 \cdot 62 \mathrm{~b}$ & $3 \cdot 62_{\text {c-e }}$ & $5 \cdot 15_{\mathrm{jk}}$ & $3 \cdot 89_{\mathrm{f}-\mathrm{m}}$ \\
\hline 9 & NGC53 & $73 \cdot 00_{\mathrm{d}-\mathrm{h}}$ & $156 \cdot 57_{b-h}$ & $18 \cdot 57_{b-h}$ & $5 \cdot 50_{h-j}$ & $14 \cdot 49_{\mathrm{a}-\mathrm{e}}$ & $4 \cdot 45 \mathrm{a}$ & $7 \cdot 79_{\mathrm{bc}}$ & $3 \cdot 37_{\mathrm{d}-\mathrm{g}}$ & $11 \cdot 57_{\mathrm{d}-\mathrm{j}}$ & $3 \cdot 30_{\mathrm{g}-\mathrm{n}}$ \\
\hline 10 & NGC40 & $72 \cdot 61_{\mathrm{d}-\mathrm{h}}$ & $192 \cdot 38_{\mathrm{ab}}$ & $20 \cdot 08_{b-f}$ & $11 \cdot 21_{b-d}$ & $14 \cdot 33_{\mathrm{a}-\mathrm{f}}$ & $3 \cdot 05_{b c}$ & $7 \cdot 77_{\mathrm{bc}}$ & $3 \cdot 34_{\mathrm{d}-\mathrm{g}}$ & $6 \cdot 20_{\mathrm{i}-\mathrm{k}}$ & $10 \cdot 87 \mathrm{a}$ \\
\hline 11 & NGC29 & $72 \cdot 42 \mathrm{~d}-\mathrm{h}$ & $125 \cdot 32 \mathrm{~h}-\mathrm{k}$ & $15 \cdot 89_{\mathrm{e}-\mathrm{i}}$ & $9 \cdot 69_{\mathrm{b}-\mathrm{f}}$ & $11 \cdot 72_{\mathrm{f}-\mathrm{l}}$ & $3 \cdot 19_{b c}$ & $8.92 b$ & $3 \cdot 25 \mathrm{~d}-\mathrm{h}$ & $6 \cdot 19_{\mathrm{i}-\mathrm{k}}$ & $3 \cdot 18_{\mathrm{g}-\mathrm{n}}$ \\
\hline 12 & NGC45 & $72 \cdot 30_{\mathrm{d}-\mathrm{h}}$ & $143 \cdot 36_{\mathrm{d}-\mathrm{j}}$ & $16 \cdot 76_{d-i}$ & $11 \cdot 38_{b-d}$ & $14 \cdot 34 \mathrm{a}-\mathrm{f}$ & $3 \cdot 17_{b c}$ & $8 \cdot 29_{b c}$ & $3 \cdot 24_{d-h}$ & $9 \cdot 89_{\mathrm{e}-\mathrm{k}}$ & $3 \cdot 78_{\mathrm{f}-\mathrm{n}}$ \\
\hline 13 & NGC26 & $71 \cdot 73_{\mathrm{d}-\mathrm{i}}$ & $136 \cdot 45_{\mathrm{f}-\mathrm{k}}$ & $11 \cdot 91_{\mathrm{i}}$ & $6 \cdot 00_{g-j}$ & $13 \cdot 45 \mathrm{~b}-\mathrm{i}$ & $3 \cdot 22_{b c}$ & $7 \cdot 46_{b c}$ & $3 \cdot 19_{\mathrm{e}-\mathrm{h}}$ & $7 \cdot 33_{\mathrm{g}-\mathrm{k}}$ & $2 \cdot 93_{\mathrm{i}-\mathrm{n}}$ \\
\hline 14 & NGC51 & $71 \cdot 71_{\mathrm{d}-\mathrm{i}}$ & $188 \cdot 61_{\mathrm{a}-\mathrm{c}}$ & $20 \cdot 94 \mathrm{~b}-\mathrm{e}$ & $6 \cdot 29_{f-j}$ & $11 \cdot 23 \mathrm{~h}-\mathrm{l}$ & $3 \cdot 20 \mathrm{bc}$ & $8 \cdot 35 \mathrm{bc}$ & $2 \cdot 88_{\mathrm{gh}}$ & $7 \cdot 76_{\mathrm{g}-\mathrm{k}}$ & $2 \cdot 01_{\mathrm{m}-\mathrm{o}}$ \\
\hline 15 & NGC50 & $70 \cdot 27_{\mathrm{d}-\mathrm{j}}$ & $125 \cdot 52_{\mathrm{h}-\mathrm{k}}$ & $16 \cdot 26_{\mathrm{e}-\mathrm{i}}$ & $8 \cdot 92_{\mathrm{c}-\mathrm{h}}$ & $11 \cdot 47_{\mathrm{g}-1}$ & $3 \cdot 15 \mathrm{bc}$ & $12 \cdot 92_{\mathrm{a}}$ & $3 \cdot 36_{\mathrm{d}-\mathrm{g}}$ & $11 \cdot 00_{\mathrm{d}-\mathrm{k}}$ & $3 \cdot 10_{\mathrm{h}-\mathrm{n}}$ \\
\hline 16 & NGC54 & $68.96_{d-j}$ & $149 \cdot 86_{c-i}$ & $20 \cdot 57_{\text {b-e }}$ & $6 \cdot 31_{f-j}$ & $15 \cdot 01_{\mathrm{a}-\mathrm{c}}$ & $3 \cdot 59_{\mathrm{a}-\mathrm{c}}$ & $9 \cdot 02 b$ & $3 \cdot 91_{b c}$ & $6 \cdot 83_{\mathrm{h}-\mathrm{k}}$ & $4 \cdot 03_{\mathrm{f}-\mathrm{m}}$ \\
\hline 17 & NGC8 & $68 \cdot 50_{d-j}$ & $123 \cdot 58_{\mathrm{h}-\mathrm{k}}$ & $16 \cdot 00_{\mathrm{e}-\mathrm{i}}$ & $7 \cdot 50_{\mathrm{e}-\mathrm{i}}$ & $12 \cdot 51_{\mathrm{c}-\mathrm{k}}$ & $3 \cdot 58_{\mathrm{a}-\mathrm{c}}$ & $7 \cdot 34 \mathrm{bc}$ & $3 \cdot 00_{\mathrm{g}-\mathrm{h}}$ & $5 \cdot 75_{\mathrm{i}-\mathrm{k}}$ & $1 \cdot 77_{\text {no }}$ \\
\hline 18 & NGC24 & $68 \cdot 39_{d-j}$ & $146 \cdot 02 \mathrm{~d}-\mathrm{j}$ & $17 \cdot 61_{\mathrm{d}-\mathrm{h}}$ & $7 \cdot 07 e-j$ & $13 \cdot 42 \mathrm{~b}-\mathrm{i}$ & $3 \cdot 29 \mathrm{bc}$ & $8 \cdot 42 \mathrm{bc}$ & $3 \cdot 64_{c-e}$ & $11 \cdot 45 \mathrm{~d}-\mathrm{j}$ & $2 \cdot 36 \mathrm{l}-\mathrm{o}$ \\
\hline
\end{tabular}

Plant Height (PH), Number of Leaf (NL), Number of Branches (NB), Harvestable Point (HP), Stem Diameter (SD), Internodes Length (IL), Leaf Length (LL), Leaf Breadth (LB), Number of Flower Bud (NFB) and Yield/plant 
Table 2 Mean performance of 34 tea genotypes evaluated for 10 agronomic characters in Mambilla continued....

\begin{tabular}{|c|c|c|c|c|c|c|c|c|c|c|c|}
\hline $\mathrm{S} / \mathrm{N}$ & Genotypes & $\begin{array}{l}\text { Plant } \\
\text { Height }\end{array}$ & $\begin{array}{l}\text { No of } \\
\text { Leaves }\end{array}$ & $\begin{array}{l}\text { No of } \\
\text { Branches }\end{array}$ & $\begin{array}{l}\text { Harvestable } \\
\text { Point }\end{array}$ & $\begin{array}{l}\text { Stem } \\
\text { Diameter }\end{array}$ & $\begin{array}{l}\text { Internodes } \\
\text { Length }\end{array}$ & $\begin{array}{l}\text { Leaf } \\
\text { Length }\end{array}$ & $\begin{array}{l}\text { Leaf } \\
\text { Breadth }\end{array}$ & $\begin{array}{l}\text { No of } \\
\text { Flower Bud }\end{array}$ & Yield/Plant(g) \\
\hline 19 & C318 & $67 \cdot 53_{\mathrm{d}-\mathrm{j}}$ & $141 \cdot 85_{e-j}$ & $17 \cdot 35_{\mathrm{d}-\mathrm{i}}$ & $7 \cdot 79_{\mathrm{e}-\mathrm{i}}$ & $14.99_{\mathrm{a}-\mathrm{c}}$ & $3 \cdot 45_{a-c}$ & $8 \cdot 74 \mathrm{bc}$ & $3 \cdot 97 \mathrm{bc}$ & $7 \cdot 40_{\mathrm{g}-\mathrm{k}}$ & $4 \cdot 85_{\mathrm{e}-\mathrm{i}}$ \\
\hline 20 & NGC12 & $66 \cdot 51_{e-j}$ & $113 \cdot 85_{\mathrm{i}-\mathrm{k}}$ & $14 \cdot 81_{\mathrm{f}-\mathrm{i}}$ & $3.95_{\mathrm{jk}}$ & $10 \cdot 44_{j-1}$ & $3 \cdot 25 b c$ & $8.06 \mathrm{bc}$ & $3 \cdot 29_{d-h}$ & $12 \cdot 28_{\mathrm{c}-\mathrm{j}}$ & $1 \cdot 78_{\text {no }}$ \\
\hline 21 & NGC42 & $66 \cdot 48$ e-j & $113 \cdot 76_{\mathrm{i}-\mathrm{k}}$ & $15 \cdot 96 \mathrm{e}-\mathrm{i}$ & $9 \cdot 53 \mathrm{~b}-\mathrm{f}$ & $12 \cdot 37_{\mathrm{c}-\mathrm{k}}$ & $3 \cdot 42 b c$ & $8 \cdot 05 b c$ & $3 \cdot 59_{c-e}$ & $13 \cdot 44_{c-i}$ & $5 \cdot 54 \mathrm{~d}-\mathrm{f}$ \\
\hline 22 & NGC23 & $66 \cdot 29_{e-j}$ & $153 \cdot 54_{b-i}$ & $19 \cdot 44_{b-f}$ & $6 \cdot 55_{\mathrm{f}-\mathrm{j}}$ & $12 \cdot 98_{b-j}$ & $3 \cdot 07 \mathrm{bc}$ & $8 \cdot 10_{b c}$ & $3 \cdot 22_{\mathrm{d}-\mathrm{h}}$ & $12 \cdot 42_{c-j}$ & $2 \cdot 50_{\mathrm{k}-\mathrm{o}}$ \\
\hline 23 & NGC37 & $63 \cdot 98_{\mathrm{f}-\mathrm{k}}$ & $181 \cdot 54 a-e$ & $21 \cdot 28_{b-e}$ & $9 \cdot 47_{b-g}$ & $12 \cdot 17_{\mathrm{d}-\mathrm{k}}$ & $3 \cdot 16_{b c}$ & $8 \cdot 51_{b c}$ & $3 \cdot 18_{\mathrm{e}-\mathrm{h}}$ & $30 \cdot 90_{\mathrm{b}}$ & $4 \cdot 63_{e-j}$ \\
\hline 24 & NGC38 & $63 \cdot 21_{\mathrm{f}-\mathrm{k}}$ & $205 \cdot 30_{a}$ & $20 \cdot 75$ b-e & $5 \cdot 14_{\mathrm{i}-\mathrm{k}}$ & $13 \cdot 73 \mathrm{~b}-\mathrm{h}$ & $3 \cdot 20 \mathrm{bc}$ & $7 \cdot 88 \mathrm{bc}$ & $2 \cdot 79_{\mathrm{h}}$ & $31 \cdot 72 b$ & $2 \cdot 36 \mathrm{l}-\mathrm{o}$ \\
\hline 25 & NGC15 & $62 \cdot 73_{\mathrm{f}-\mathrm{l}}$ & $138 \cdot 48 \mathrm{f}-\mathrm{k}$ & $16.96 \mathrm{~d}-\mathrm{i}$ & $6 \cdot 88_{e-j}$ & $12 \cdot 97 \mathrm{b-j}$ & $3 \cdot 12 \mathrm{bc}$ & $8 \cdot 48 \mathrm{bc}$ & $3 \cdot 27_{\mathrm{d}-\mathrm{h}}$ & $5 \cdot 78_{\mathrm{i}-\mathrm{k}}$ & $3 \cdot 04_{i-n}$ \\
\hline 26 & NGC41 & $62 \cdot 41_{\mathrm{g}-1}$ & $154 \cdot 55_{\mathrm{b}-\mathrm{i}}$ & $19 \cdot 07_{\mathrm{b}-\mathrm{g}}$ & $9 \cdot 56_{b-f}$ & $12 \cdot 51_{\mathrm{c}-\mathrm{k}}$ & $3 \cdot 20_{b c}$ & $8 \cdot 82_{b}$ & $3 \cdot 73_{b-d}$ & $3 \cdot 44_{k}$ & $6 \cdot 12_{\mathrm{de}}$ \\
\hline 27 & NGC47 & $62 \cdot 23_{\mathrm{g}-1}$ & $128 \cdot 88_{\mathrm{g}-\mathrm{k}}$ & $14 \cdot 43_{\mathrm{f}-\mathrm{i}}$ & $7 \cdot 20_{e-j}$ & $12 \cdot 93_{\mathrm{c}-\mathrm{j}}$ & $3 \cdot 58_{\mathrm{a}-\mathrm{c}}$ & $8 \cdot 86_{b}$ & $3 \cdot 32_{\mathrm{d}-\mathrm{g}}$ & $13 \cdot 00_{c-j}$ & $3 \cdot 00_{\mathrm{i}-\mathrm{n}}$ \\
\hline 28 & NGC32 & $60 \cdot 46 \mathrm{~h}-1$ & $146 \cdot 84_{d-j}$ & $20 \cdot 80$ b-e & $12 \cdot 58_{\mathrm{ab}}$ & $11 \cdot 24 \mathrm{~h}-\mathrm{I}$ & $2 \cdot 89 \mathrm{bc}$ & $7 \cdot 75 \mathrm{bc}$ & $3 \cdot 32 \mathrm{~d}-\mathrm{g}$ & $17 \cdot 21_{\text {c-e }}$ & $2 \cdot 61_{\mathrm{j}-\mathrm{o}}$ \\
\hline 29 & NGC6 & $59 \cdot 00_{\mathrm{i}-\mathrm{m}}$ & $106 \cdot 09_{\mathrm{jk}}$ & $12 \cdot 93_{\mathrm{hi}}$ & $8 \cdot 39_{\mathrm{c}-\mathrm{i}}$ & $9 \cdot 87_{\mathrm{kl}}$ & $3 \cdot 29 \mathrm{bc}$ & $7 \cdot 34 \mathrm{bc}$ & $3 \cdot 16 \mathrm{e}-\mathrm{h}$ & $5 \cdot 18_{\mathrm{jk}}$ & $4 \cdot 36 \mathrm{e}-1$ \\
\hline 30 & NGC46 & $58 \cdot 39_{\mathrm{j}-\mathrm{m}}$ & $150 \cdot 21_{c-i}$ & $16 \cdot 31_{\mathrm{e}-\mathrm{i}}$ & $9 \cdot 05_{c-g}$ & $11 \cdot 94_{\mathrm{d}-\mathrm{k}}$ & $3 \cdot 38 \mathrm{bc}$ & $7 \cdot 86 \mathrm{bc}$ & $3 \cdot 30_{\mathrm{d}-\mathrm{g}}$ & $12 \cdot 24_{\mathrm{c}-\mathrm{j}}$ & $4 \cdot 57_{\mathrm{e}-\mathrm{k}}$ \\
\hline 31 & NGC22 & $52 \cdot 51_{\mathrm{k}-\mathrm{m}}$ & $105 \cdot 43_{\mathrm{jk}}$ & $13 \cdot 67_{\mathrm{g}-\mathrm{i}}$ & $6 \cdot 91_{e-j}$ & $11 \cdot 80_{\mathrm{e}-\mathrm{l}}$ & $2 \cdot 88 \mathrm{bc}$ & $7 \cdot 91 \mathrm{bc}$ & $2 \cdot 96 \mathrm{gh}$ & $9 \cdot 85 \mathrm{e}-\mathrm{k}$ & $2 \cdot 24_{\mathrm{m}-\mathrm{o}}$ \\
\hline 32 & NGC48 & $50 \cdot 65 \mathrm{~lm}$ & $134 \cdot 56 \mathrm{f}-\mathrm{k}$ & $17 \cdot 33 \mathrm{~d}-\mathrm{i}$ & $8 \cdot 70_{c-h}$ & $13 \cdot 17_{b-j}$ & $2 \cdot 73_{c}$ & $8 \cdot 72 \mathrm{bc}$ & $3 \cdot 51_{c-f}$ & $6 \cdot 13 \mathrm{i}-\mathrm{k}$ & $4 \cdot 91_{\mathrm{e}-\mathrm{i}}$ \\
\hline 33 & NGC17 & $47 \cdot 80_{\mathrm{m}}$ & $97 \cdot 48_{\mathrm{k}}$ & $15 \cdot 58_{\text {e-i }}$ & $6 \cdot 65_{e-j}$ & $10 \cdot 84_{\mathrm{i}-\mathrm{l}}$ & $2 \cdot 91_{b c}$ & $8 \cdot 23 \mathrm{bc}$ & $3 \cdot 06_{\mathrm{f}-\mathrm{h}}$ & $14 \cdot 71_{\mathrm{c}-\mathrm{g}}$ & $3 \cdot 23_{g-n}$ \\
\hline 34 & NGC55 & $34 \cdot 26_{n}$ & $43 \cdot 18$ l & $6 \cdot 29_{j}$ & $2 \cdot 40_{\mathrm{k}}$ & $9 \cdot 28_{1}$ & $2 \cdot 79_{\mathrm{bc}}$ & $5 \cdot 88_{c}$ & $3 \cdot 18_{\mathrm{e}-\mathrm{h}}$ & $9 \cdot 10_{\mathrm{f}-\mathrm{k}}$ & $0 \cdot 90_{\text {o }}$ \\
\hline
\end{tabular}

Means with the same letter along the column are not significantly different at 5\% level of probability, using DMRT. Plant Height (PH), Number of Leaf (NL), Number of Branches (NB), Harvestable Point (HP), Stem Diameter (SD), Internodes Length (IL), Leaf Length (LL), Leaf Breadth (LB), Number of Flower Bud (NFB) and Yield/plant 
World Journal of Advanced Research and Reviews, 2021, 09(01), 050-061

Table 3 Mean separation of protein, crude fibre and caffeine among 34 tea genotypes in 2 environments

\begin{tabular}{|c|c|c|c|c|}
\hline$S / N$ & Genotype & Protein & Crude Fibre & Caffeine \\
\hline 1 & NGC38 & $22 \cdot 74 a$ & $9 \cdot 39 h-j$ & $2 \cdot 34 d-h$ \\
\hline 2 & NGC 19 & $22 \cdot 51 \mathrm{ab}$ & $9 \cdot 39 h-j$ & $2 \cdot 29 e-j$ \\
\hline 3 & NGC 18 & $22 \cdot 18 \mathrm{bc}$ & $9 \cdot 21 \mathrm{j}$ & $2 \cdot 53 a b$ \\
\hline 4 & C 143 & $22 \cdot 15 b c$ & $9 \cdot 67 b-g$ & $2 \cdot 41 b-e$ \\
\hline 5 & NGC 17 & $22 \cdot 13 b-d$ & $9 \cdot 51 d-i$ & $2 \cdot 39 b-f$ \\
\hline 6 & NGC 29 & $22 \cdot 11 b-d$ & $9 \cdot 45 f-i$ & $2 \cdot 33 d-h$ \\
\hline 7 & C 357 & $22 \cdot 08 b-d$ & $9 \cdot 51 d-i$ & $2 \cdot 39 b-f$ \\
\hline 8 & NGC 13 & $22 \cdot 07 b-d$ & $9 \cdot 70 b-f$ & $2 \cdot 15 \mathrm{jk}$ \\
\hline 9 & NGC 54 & $22 \cdot 03 b-e$ & $9 \cdot 29 \mathrm{ij}$ & $2 \cdot 26 \mathrm{e}-\mathrm{k}$ \\
\hline 10 & NGC 32 & $21 \cdot 98 b-e$ & $9 \cdot 70 b-f$ & $2 \cdot 25 \mathrm{e}-\mathrm{k}$ \\
\hline 11 & NGC 26 & $21 \cdot 98 b-e$ & $9 \cdot 82 \mathrm{bc}$ & $2 \cdot 19 \mathrm{~h}-\mathrm{k}$ \\
\hline 12 & NGC 46 & $21.95 b-e$ & $9 \cdot 44 g-j$ & $2 \cdot 09 \mathrm{k}$ \\
\hline 13 & NGC 45 & $21 \cdot 95 b-e$ & $9 \cdot 59 \mathrm{c}-\mathrm{g}$ & $2 \cdot 25 \mathrm{e}-\mathrm{k}$ \\
\hline 14 & NGC 27 & $21 \cdot 83 c-f$ & $9 \cdot 53 d-i$ & $2 \cdot 23 \mathrm{f}-\mathrm{k}$ \\
\hline 15 & NGC 37 & $21 \cdot 58 \mathrm{c}-\mathrm{g}$ & $9 \cdot 73 b-d$ & $2 \cdot 11 \mathrm{k}$ \\
\hline 16 & NGC 41 & $21 \cdot 53 \mathrm{~d}-\mathrm{h}$ & $9 \cdot 71 \mathrm{~b}-\mathrm{e}$ & $2 \cdot 17 \mathrm{i}-\mathrm{k}$ \\
\hline 17 & NGC 24 & $21 \cdot 53 \mathrm{~d}-\mathrm{h}$ & $9 \cdot 29 \mathrm{ij}$ & $2 \cdot 25 \mathrm{e}-\mathrm{k}$ \\
\hline 18 & NGC 47 & $21 \cdot 51 \mathrm{~d}-\mathrm{h}$ & $9 \cdot 62 b-h$ & $2 \cdot 13 \mathrm{jk}$ \\
\hline 19 & NGC 22 & $21 \cdot 45 \mathrm{e}-\mathrm{i}$ & $9 \cdot 81 b c$ & $2 \cdot 21 \mathrm{~g}-\mathrm{k}$ \\
\hline 20 & NGC 25 & $21 \cdot 33 f-j$ & $9 \cdot 61 c-h$ & $2 \cdot 23 \mathrm{f}-\mathrm{k}$ \\
\hline 21 & NGC 53 & $21 \cdot 28 f-j$ & $9 \cdot 49 \mathrm{~d}-\mathrm{i}$ & $2 \cdot 51 \mathrm{a}-\mathrm{c}$ \\
\hline 22 & NGC 55 & $21 \cdot 21 g-j$ & $9 \cdot 66 \mathrm{~b}-\mathrm{g}$ & $2 \cdot 34 c-h$ \\
\hline 23 & C318 & $21 \cdot 08 \mathrm{~g}-\mathrm{k}$ & $9 \cdot 86 b$ & $2 \cdot 12 \mathrm{k}$ \\
\hline 24 & NGC 6 & $21 \cdot 01 \mathrm{~g}-\mathrm{k}$ & $9 \cdot 47 e-i$ & $2 \cdot 15 \mathrm{jk}$ \\
\hline 25 & NGC 12 & $21 \cdot 00 \mathrm{~g}-\mathrm{k}$ & $9 \cdot 43 g-j$ & $2 \cdot 15 \mathrm{jk}$ \\
\hline 26 & NGC 15 & $20 \cdot 95 \mathrm{~h}-\mathrm{k}$ & $9 \cdot 47 e-i$ & $2 \cdot 46 b-d$ \\
\hline 27 & NGC 51 & $20 \cdot 85 \mathrm{i}-\mathrm{k}$ & $9 \cdot 39 h-j$ & $2 \cdot 49 b-d$ \\
\hline 28 & NGC 48 & $20 \cdot 80 \mathrm{jk}$ & $9 \cdot 32 \mathrm{ij}$ & $2 \cdot 37 \mathrm{~b}-\mathrm{g}$ \\
\hline 29 & NGC 40 & $20 \cdot 61 \mathrm{k}$ & $10 \cdot 16 a$ & $2 \cdot 65 a$ \\
\hline 30 & NGC 49 & $20 \cdot 55 \mathrm{k}$ & $9 \cdot 28 \mathrm{ij}$ & $2 \cdot 52 \mathrm{ab}$ \\
\hline 31 & NGC 50 & $18 \cdot 951$ & $8 \cdot 55 \mathrm{k}$ & $2 \cdot 33 d-i$ \\
\hline 32 & NGC 8 & $18 \cdot 831$ & $8 \cdot 41 \mathrm{k}$ & $1 \cdot 371$ \\
\hline 33 & NGC23 & $20.55 \mathrm{k}$ & $9.86 \mathrm{~b}$ & $2.47 \mathrm{~b}-\mathrm{d}$ \\
\hline 34 & NGC42 & $21.11 \mathrm{bd}$ & $9.81 b c$ & $2.14 \mathrm{jk}$ \\
\hline
\end{tabular}


The Principal Component Analysis (PCA) bi-plot shows the diversity existing among 34 tea g genotypes is presented in figure 1, 2, and 3. The PCA 2 plotted against PCA1 (Figure 1). PCA 1explained $37.23 \%$ of the total variation, while PC 2 accounted for $15.48 \%$ and both axes accounted for $52.72 \%$ of the diversity exists among the 34 tea genotypes. The biplot (Figure 1) showed that genotypes C357, NGC 55, NGC 8, NGC 42 and C143 were quite distinct from other tea genotypes that were clustered together at the centre of the bi-plot.

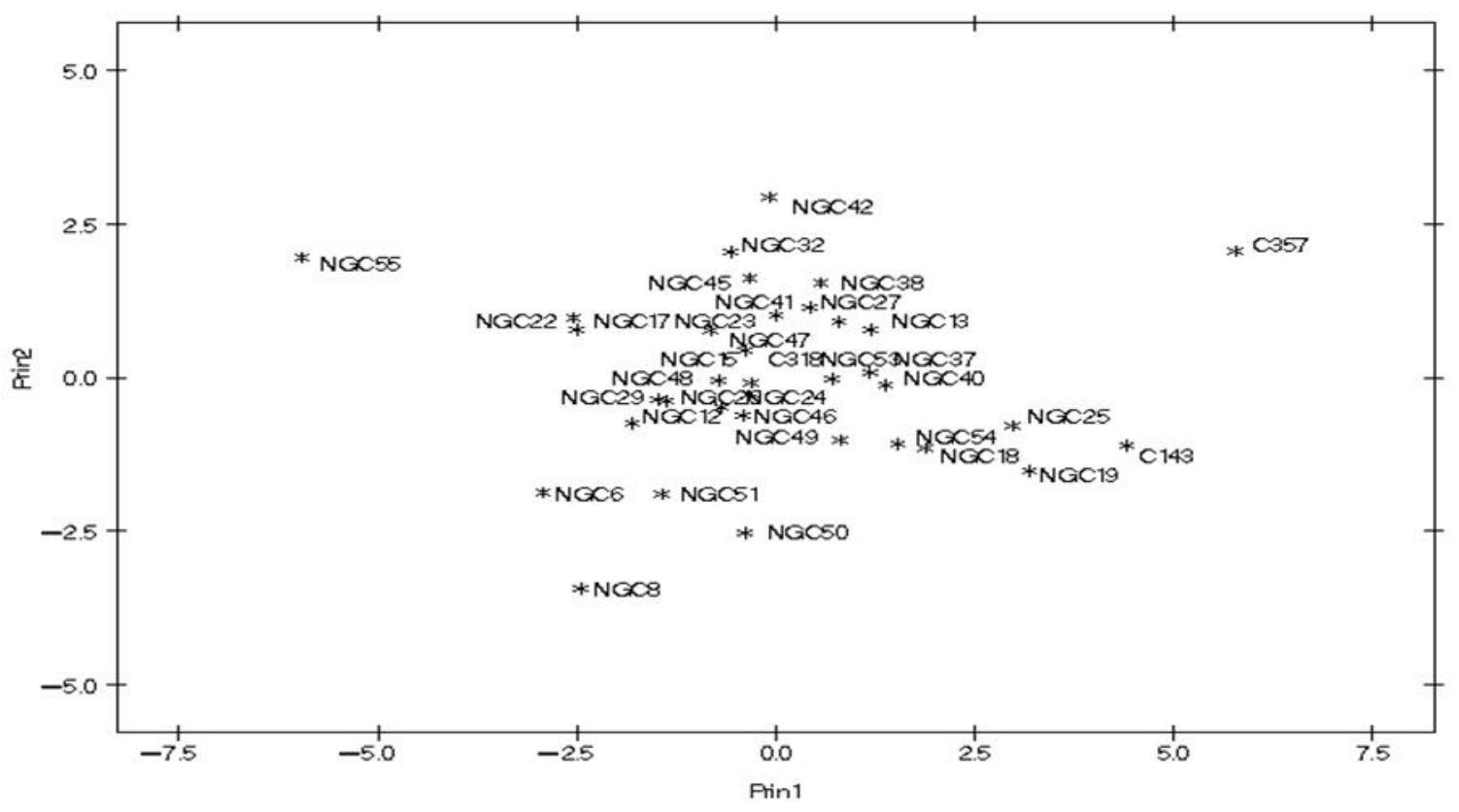

Figure 1 PC1 And PC2 bi plot of variation existing among 34 tea genotypes

The dendogram generated from single linkages cluster analysis (SLCA) using morphological characters is presented in Figure 2. The dendrogram illustrates the relationship among 34 tea genotypes. At a minimum 0.00 level of similarity, all genotypes were distinct from one another while at 1.25 level of similarity, all the genotypes had formed a single cluster. The dendogram showed that the first cluster was observed between NGC29 and NGC 47 at 0.08 level of similarity. At 0.50 level of similarity the dendogram revealed 5 distinct groups. Group I had highest number of genotypes (28), groups II and group V had only one member each C357 and NGC 55 respectively. Groups III and IV had two members each. Group III consist of NGC 19 NGC 25 and while group IV had NGC 37 and NGC 38.

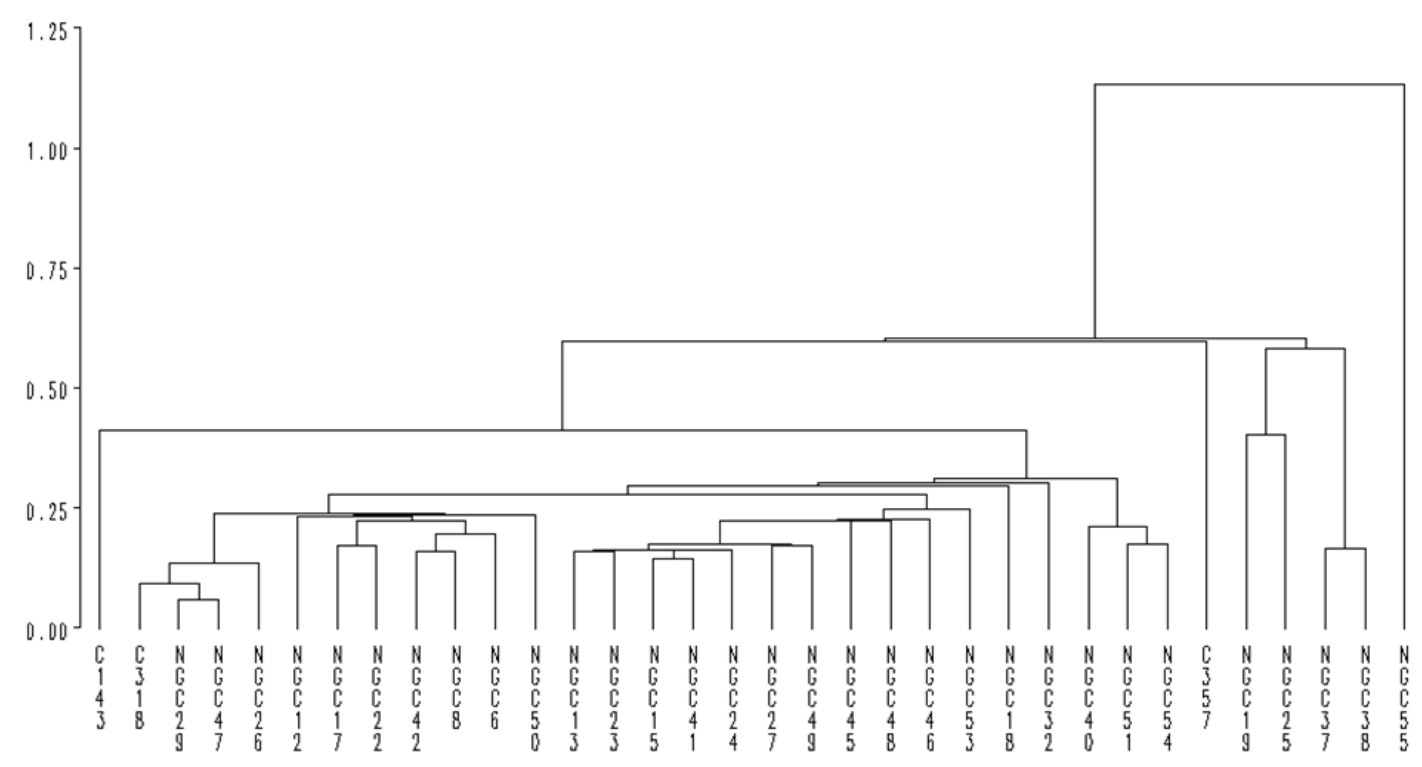

Figure 2 Dendogram generated from Single Linkage Cluster Analysis (SLCA) on yield of 34 tea 
Table 4 shows the results of principal component analysis of 34 tea genotypes tried. Only three of the 10 principal components had eigen values greater than 1 and the first 5 axes accounted for $37.23 \%, 15.48 \%, 10.75 \%, 0.09 \%$ and $0.07 \%$ of the total variation individually and $79.41 \%$ altogether. The first principal component was loaded largely by Plant height (PH) 0.39, stem diameter (SD) $0 \cdot 39$, Number of leaves (NL) $0 \cdot 38$, Number of branches (NB) $0 \cdot 37$, Harvestable point (HP) 0.31 , and leave breadth (LB) 0.30 . Axis 2 was loaded with protein 0.45 , crude fibre 0.57 and caffeine 0.45 . However, axis 3 was largely loaded with harvestable point (HP), Internodes length (IL) and yield/plant with value of $-0.44,0.61$ and -0.46 respectively. The relative discriminating power of the principal axes as indicated by eigen values was 4.84 for axis one and 0.69 for axis five.

Table 4 Principal component analysis showing the contribution (factor scores), eigen values and percentage total variance accounted for by the first five principal component axes of each character of the 34 tea genotypes in 3 environments.

\begin{tabular}{|l|l|l|l|l|l|}
\hline Character & Pc1 & Pc2 & Pc3 & Pc4 & Pc5 \\
\hline PH & 0.39 & -0.22 & 0.15 & 0.01 & -0.07 \\
\hline NL & 0.38 & -0.15 & -0.10 & -0.28 & 0.17 \\
\hline NB & 0.37 & -0.16 & -0.06 & -0.21 & 0.09 \\
\hline HP & 0.31 & 0.08 & -0.44 & 0.17 & 0.02 \\
\hline SD & 0.39 & 0.08 & 0.15 & -0.23 & -0.18 \\
\hline IL & 0.15 & -0.14 & 0.61 & 0.60 & -0.26 \\
\hline LL & 0.21 & -0.19 & 0.19 & 0.36 & 0.29 \\
\hline LB & 0.30 & 0.17 & 0.14 & -0.17 & -0.38 \\
\hline NFB & 0.22 & 0.17 & -0.05 & -0.05 & 0.58 \\
\hline Yield/Plt & 0.28 & -0.09 & -0.46 & -0.05 & -0.28 \\
\hline Protein & 0.07 & 0.49 & 0.08 & -0.45 & 0.04 \\
\hline Crude Fibre & 0.06 & 0.57 & -0.11 & 0.19 & -0.22 \\
\hline Caffeine & 0.14 & 0.45 & 0.25 & 0.18 & 0.28 \\
\hline Eigen value & 4.84 & 2.01 & 1.40 & 0.94 & 0.69 \\
\hline \% Variance & 37.23 & 15.48 & 10.75 & 0.09 & 0.07 \\
\hline Cumulative & 37.23 & 52.72 & 63.47 & 72.17 & 79.41 \\
\hline
\end{tabular}

Plant Height (PH), Number of Leaf (NL), Number of Branches (NB), Stem Diameter (SD), harvestable points (HP), Internodes Length (IL), Leaf Length (LL), Leaf Breadth (LB), Number of Flower Bud (NFB) and Yield/plt.

Major characteristic pattern of tea genotypes by FASTCLUS technique is presented in Table 5. The 34 genotypes were partitioned into seven distinct groups by the FASTCLUS technique. Group I had only one genotype while group III and VII have three genotypes each. Group II had six genotypes while group VI and IV had the highest number of genotypes, ten and nine genotypes respectively. Group V contained only two genotypes. Group V with genotypes NGC 19 and NGC 25 were characterized by high plant height, number of leaves, number of branches, harvestable point as well as high yield. The highest caffeine and protein were observed in group III with genotypes NGC 38, C357 and NGC37. 
Table 5 Characteristic pattern of seven groups of tea genotypes by FASTCLUS techniques

\begin{tabular}{|c|c|c|c|c|c|c|c|}
\hline & Group I & Group II & Group III & Group IV & Group V & Group VI & Group VII \\
\hline & NGC55 & $\begin{array}{l}\text { NGC40, NGC51, } \\
\text { NGC53, NGC54, } \\
\text { C143, NGC18 }\end{array}$ & $\begin{array}{l}\text { NGC 38, C357, } \\
\text { NGC37 }\end{array}$ & $\begin{array}{l}\text { NGC12, NGC26, } \\
\text { NGC32, NGC29, } \\
\text { NGC47, NGC50, }\end{array}$ & NGC19, NGC25 & $\begin{array}{lr}\text { NGC13, NGC 15, } \\
\text { NGC23, } \\
\text { NGC27, }\end{array}$ & $\begin{array}{l}\text { NGC22, NGC6, } \\
\text { NGC17 }\end{array}$ \\
\hline Plant height $(\mathrm{cm})$ & 30.30 & $81.59(12.29)$ & $76.68(17.84)$ & $64.79(5.57)$ & $91.44(9.25)$ & $71.50(10.42)$ & $52.89(7.13)$ \\
\hline Number of leaves & 35.78 & $202.57(8.78)$ & $219.55(9.62)$ & $132.80(8.17)$ & $252.60(1.15)$ & $167.30(9.73)$ & $107.39(6.84)$ \\
\hline Number of branches & 6.39 & $29.26(5.74)$ & $25.11(1.75)$ & $18.57(3.14)$ & $30.27(1.75)$ & $21.90(1.93)$ & $15.60(1.89)$ \\
\hline Harvestable points & 2.50 & $10.28(4.27)$ & $11.57(4.94)$ & $9.15(3.06)$ & $12.60(5.28)$ & $9.60(1.74)$ & $7.89(0.12)$ \\
\hline Stem Diameter & 9.71 & $13.54(1.53)$ & $14.22(2.38)$ & $11.94(0.94)$ & $14.36(1.85)$ & $12.84(0.57)$ & $10.88(0.01)$ \\
\hline Internodes length & 2.86 & $3.54(0.42)$ & $3.38(0.28)$ & $3.34(0.29)$ & $3.15(0.16)$ & $3.39(0.34)$ & $3.09(0.36)$ \\
\hline Leaf length & 5.35 & $8.78(0.99)$ & $9.21(1.23)$ & 8.17 (2.21) & $9.20(0.10)$ & $8.78(0.74)$ & $8.34(0.59)$ \\
\hline Leaf Breadth & 3.26 & $3.42(0.47)$ & $3.77(1.33)$ & $3.42(0.29)$ & $3.86(0.46)$ & $3.57(0.22)$ & $3.18(0.05)$ \\
\hline No of flower buds & 2.06 & $8.95(4.14)$ & $45.78(2.94)$ & $9.93(6.49)$ & $15.14(8.80)$ & $9.39(4.09)$ & $6.42(2.16)$ \\
\hline Yield & 0.97 & 5.40 (2.99) & $4.79(1.56)$ & $3.26(1.26)$ & 8.19 (3.49) & 3.79 (1.42) & $3.34(1.37)$ \\
\hline Protein & 20.85 & $20.71(0.63)$ & $21.21(0.55)$ & $20.59(1.15)$ & $20.52(0.07)$ & $20.68(0.65)$ & $20.57(0.77)$ \\
\hline Crude fibre & 9.34 & $8.89(0.41)$ & $9.34(0.29)$ & $9.80(0.48)$ & $8.95(0.25)$ & $9.35(0.41)$ & $8.90(0.31)$ \\
\hline Caffeine & 2.16 & $2.22(0.22)$ & $2.40(0.47)$ & $2.10(0.37)$ & $2.05(0.02)$ & $2.24(0.24)$ & $2.11(0.43)$ \\
\hline
\end{tabular}




\section{Discussion}

The success of any breeding programme depends upon the variations in the genetic resource. The greater the genetic variability, the better is the chances for success to be achieved through selection. The significant variations among the 34 tea genotypes with respects to the 22 traits that were measured indicated the wealth of genetic diversity present within the genotypes and also gave a high prospect in varietal improvement of tea. Earlier studies on tea showed the presence of wide range of diversity in both quantitative and qualitative traits [13] and [16].

The significant mean squares obtained from the analysis of variance for the genotypes indicated that at least two genotypes were different, resulting to the possibility of selection. Earlier studies on tea genetic classification using agromorphological traits such as leaf length, leaf breadth, flower bud and yield parameter had been reported to be a great importance in distinguishing genotypes in tea [7]. However, the exploitation of these plant traits in the breeding programme of tea species will be enhanced by understanding the extent of variability existing for these traits and genetic components that governed the expression. The result of the principal component analysis confirms these patterns of character - co variation among the genotypes studied. It also identified the characters that contributed most to the variation within a group of entries [17]. The biological meaning of the principal component can be accessed from the contribution of the different variables to each principal component according to the eigen vectors [14]. The result of the principal component analysis revealed that different characters contributed differently to the total variation as indicated by their eigen vectors as well as their weight and loading on different principal axes.

The identification of principal component analysis (PCA) of plant height, number of leaves, number of branches, harvestable point, stem diameter and yield/plant as major components of variation among the tea genotypes indicated the reliability in the use of these characters in distinguishing among tea genotypes.

The observations are in line with those of [20] who stated that character coefficient greater than 0.3 , irrespective of the arithmetic sign of the coefficient, be treated as having a large effect to be considered important in classifying the genotypes. The first four principal axes accounted for $72.17 \%$ of the total variation among the thirteen quantitative characters describing the genotypes. This confirmed the report of [4] who reported that the first three Principal components were the most important in reflecting the variation on patterns among genotypes and the characters highly associated with these should be used in differentiating genotypes along the principal component axis and the proportion of the total variation contributed by each of the different variables [23]

The FASTCLUS technique was used to sort the genotypes into seven groups with unique information about morphological characteristic were identified. The two genotypes in group V NGC19 and NGC25 can be described as high yielding genotypes while NGC55 only genotype in group I can be associated with low flowering and low yielding genotypes.

The wide range of similarity coefficient observed from the dendrogram generated by single linkage custer analysis (SLCA) revealed high variability among the tea genotypes [2] reported that cluster analysis as a tool had singular efficacy and ability to identify crop accessions with high similarity using dendrogram.

Caffeine, protein and crude fiber are major constituents in tea. The activities of caffeine and when consumed by humans draws more attention to its degree of accumulation in different tea genotypes. This substance is naturally occurring in tea leaves and many crops such as coffee, kola e.t.c. [24]

\section{Conclusion}

The current study has shown that characterization of genetic materials provide a way of evaluating germplasm in order to identify genotypes that could be further evaluated and utilized at genetic level. 34 tea genotypes were studied using thirteen agro morphological traits

The result showed that variability exists among the genotypes considered in this study as indicated by analysis of variance, PCA and Dendogram.

This study has revealed greater variability among the 34 genotypes an indication for tea genetic improvement in Nigeria. It also manifested some quality traits in terms of crude fibre, protein and caffeine content which can also be incorporated into tea improvement programme to suit the preference of consumers in Nigeria and beyond. 


\section{Compliance with ethical standards}

\section{Disclosure of conflict of interest}

All authors declare that no conflict of interest is exist.

\section{References}

[1] Adebisi MA, OJ Ariyo, OB Kehinde. Variation and correlation studies in quantitative characters in Soyabean. The Ogun J. Agric Sci. 2004; 3(1): 134-142.

[2] Aliyu B, NQ Ng, I Fawole. Inheritance of Pubescence in crosses between VignaUnguculata and V. vhomboidea. Nigeria Journal of Genetics. 2000; 15: 9-14.

[3] Aremu CO. Diversity Selection and Genotype X Environment Interaction in Cowpea (Vignaunguiculata (L.)Walp) PhD. Thesis, University of Agric. Abeokuta, Nigeria. 2005; 125.

[4] Clifford HT, Stephenson W. An Introduction to numerical Classification Academy Press, London, New York, xii. 1975; 229.

[5] Debra L, Edwards JU. Take time for tea: for health and well-being. NDSu Extension Circular. 2007.

[6] Engels J, Ramanatha M, Rao V, Brown AHD, Jackson MT. Managing Plant Genetic Diversity. IPGRI, Rome. 2002.

[7] Esan, Omolaja. Genotypic Association Path analysis and pluck quality values in Tea (Camellia simensis (L) 0. Kuntze. Trop. Agric. (Trinidad). 2002; 79(2): 1-4.

[8] Gritting RE. The Application of Ordination Techniques In: H. Bryanfand W. R. Atchley Eds. Multivariate Statistical Methods, Within Groups Covariation, Dowden, Hutchinson and Ross, Inc. Stroudsbury, Pennsylvania. 1975; 102131.

[9] Gunasekara MTK, Ramatuuga. Polyploidy in tea (Camellia sinensis L.) and its application in tea breeding; A review. Sri Lanka Journal Tea Science. 2003; 68(2): 14 - 26.

[10] Heinsworth E. Tea Production on the Mammbilla Plateau, Gongola State Nigeria.A report on the Project by the consultants for the Nig. Bev. Production C. Ltd. 1981; 26.

[11] Islam GM, Iqbal M, Quddus KG, Ali MY. Present status and future needs of tea industry in Bangladesh. Proc. Pakistian Acad. Sci. 2005; 42(4): 305-314.

[12] Islam GMR, M Iqbal, KG Quddus, MY Ali. Present and Future need of Tea Industry in Bangladesh. Proc Pakistan Acad. Sci. 2005; 42(4): 305-314.

[13] Latip SNH, Muhamad R, Manjeri G, Tan SG. Genetic variation of selected Camellia sinensis (Cultivated tea) varieties in Malaysis Based on Random Amplified Microsatellite (RAM) Markers Pertanika Journal of Tropical Agric. Sci. 2010; 33(2): 257-267.

[14] Lezzoni AF, Pritts MP. Application of Principal Component Analysis to Horticultural Research. Horticultural Science. 1991; 26(4): $334-338$.

[15] Liang C. Germplasm and Genetic Improvement of Tea Plant. Eds: Liang C., Fu-Lian Yu and Yang Y. J. China Agricultural Sciences and Technology Press ISBN: 7-80233-181-1/s.989. 2006.

[16] Nasriya M, KM Mewan, KBAG Premathilaka, DR Gimhani. Simple Sequence Repeat Markers Revealed Genetic Diversity of Old Seedlings teasin. 2011.

[17] Ogunbodede BA. Multivariate analysis of genetic diversity of kenot (Hibsiscus cannabinus L.) African Crop Science Journals. 1997; (2): 127 - 133.

[18] Omoighi LO, MF Ishiyaku, AY Kamra, SO Alabi, SG Mohammed. Genetic variability and heritability studies of some productive traits in cowpea (vigna unguiculata (L) Walp) African Journal of Biotechnology. 2006; 5(13): 11911195.

[19] Omolaja SS, Iremeren GO. Tea Improvement in Nigeria. Springer link, Global tea breeding. 2012; 323-342.

[20] Raji AA. Assessment of genetic diversity and heterosis relationship in African improved and local cassava (Manihot esculenta (rttz) germplasin Ph.D Thesis Obafemi Awolowo University, Ile-Ife, Nigeria. $2002 ; 120$. 
[21] Sealy JR. A revision of genus Camellia. Royal Horticultural Society, London. 1958; 239.

[22] Smith JSC. Genetic variability within U.S. hybrid maize: multivariate analysis of isozyme data. Crop science. 1984; 24: 1041-1046.

[23] Sneath PH, R. Sokal. Numerical Taxonomy, W. H. Freeman Son Francisco. 1973; 573.

[24] Wanyika HN, EG Gatabe, LM Gitu, EK Ngumba, CW Maritim 2010. Determination of caffeine content of Tea and instant Coffee brands found in the Kenyan Market. African Journal of Food Science. 2010; 4(6), 353-358. 